\title{
A UNIQUENESS THEOREM FOR THE SOLUTION OF A STEFAN PROBLEM
}

\author{
JIM DOUGLAS, JR.
}

1. Introduction. A Stefan problem consists of solving a parabolic differential equation subject to a boundary condition on a moving boundary the location of which is not given in advance but is determined as a portion of the problem. A number of physical problems give rise to such conditions. Among these are heat conduction involving phase change such as the melting or freezing of ice, evaporation or condensation, or recrystallization of metals. Others include various displacement processes of reservoir engineering wherein one fluid partially displaces a second (and originally resident) fluid in flowing through a porous medium.

A typical problem of this type is the determination of a function $u(x, t)$ in the region $0 \leq x \leq x(t)$, where $x(t)$ is the position of the moving boundary at time $t$, such that

$$
\begin{aligned}
\text { (a) } \frac{\partial^{2} u}{\partial x^{2}} & =f(u) \frac{\partial u}{\partial t}, & 0<x<x(t), \\
\text { (b) } \frac{\partial u}{\partial x}(0, t) & =-a, & t>0, \\
\text { (c) } u(x(t), t) & =0, & t>0, \\
\text { (d) } x(0) & =0, & t>0, \\
\text { (e) } \quad \frac{d x(t)}{d t} & =b-c \frac{\partial u}{\partial x}(x(t), t), &
\end{aligned}
$$

where $a, b$, and $c$ are non-negative constants, $f(u)>0$, and $f^{\prime}(u) \geq 0$. To the author's knowledge, neither a proof of existence nor of uniqueness of the solution of (1.1) has appeared. It is the object of this paper to demonstrate the uniqueness of the solution, deferring until later an existence argument somewhat similar to that given in [3] for the simpler case to be discussed in the next paragraph.

The case where $f(u)=1$ and $b=0$ has been treated at some length in the literature $[1 ; 3 ; 4 ; 7]$. Existence of the solution was demonstrated in each of these papers; however, no satisfactory uniqueness proof was given in any of these. (In fact, much, but not the existence proof, of [3] was based on uniqueness.) Each of the arguments

Presented to the Society, August 24, 1956; received by the editors May 21, 1956 and, in revised form, July 6, 1956. 
offered to support uniqueness made use of the following assumption: if $x_{1}(t)$ and $x_{2}(t)$ are two possible boundaries, then there exists no condensation point of the zeros of the function $\xi(t)=x_{1}(t)-x_{2}(t)$ unless $x_{1}(t) \equiv x_{2}(t)$. If $x_{1}(t)$ and $x_{2}(t)$ were analytic, this certainly would be the case. Unfortunately, no one showed this; and, indeed, since we are dealing with parabolic equations, there is no particular reason to hope that this should be true. Evans alone mentioned the above assumptions; Datzeff and Sestini apparently overlooked it.

Before turning to the proof, the author would like to acknowledge a suggestion by T. M. Gallie, Jr., which resulted in a simplification of a portion of the proof.

2. An equivalent boundary relation. So far, nothing has been said about the nature of $f(u)$ except to require that it be positive and nondecreasing. While much of the argument would go through for $f(u)$ otherwise rather unrestricted, no such lack of regularity is likely to arise in the physical problems leading to the study of the Stefan problem. In fact, most frequently the $f(u)$ actually used in a physical problem would be a polynomial or some other rather elementary function used to interpolate laboratory measurements; consequently, we shall treat $f(u)$ as if it were analytic and assume $f(0)>0$.

Let us develop a few of the properties of any solution of (1.1). First, since the boundary curve $x=x(t)$ is supposed differentiable for each $t, x(t)$ must be continuous. Next, let us prove the

Lemma 1. Let $y(t)$ be continuous, $y(0)=0$, and $y(t)>0$ for $t>0$. Let $u(x, t)$ be a solution of

$$
\begin{aligned}
u_{x x} & =f(u) u_{t}, & 0<x<y(t), \\
u_{x}(0, t) & =-a, & t>0, \\
u(y(t), t) & =0, & t>0 .
\end{aligned}
$$

Then,

$$
\begin{aligned}
0 \leq u(x, t) & \leq a(y(t)-x), & & 0<x<y(t), \\
-a \leq u_{x}(x, t) & \leq 0, & & 0<x<y(t) .
\end{aligned}
$$

Proof. As (2.2) follows from (2.3), it is sufficient to prove (2.3). We shall use a finite difference argument to obtain (2.3). Let

$$
x_{i}=i \Delta x, \quad t_{n}=n \Delta t,
$$

and

$$
g\left(x_{i}, t_{n}\right)=g_{i n} .
$$

Then, let $u_{i n}$ be the solution of the backward difference equation 


$$
\begin{aligned}
\frac{u_{i+1, n+1}-2 u_{i, n+1}+u_{i-1, n+1}}{(\Delta x)^{2}} & =f\left(u_{i n}\right) \frac{u_{i, n+1}-u_{i n}}{\Delta t}, x_{i}<y\left(t_{n+1}\right), \\
u_{1, n+1}-u_{0, n+1} & =-a \Delta x, \\
u\left(y\left(t_{n+1}\right), t_{n+1}\right) & =0 .
\end{aligned}
$$

(Note: The last second difference must be modified in an obvious way, since the last increment may be less than $\Delta x$.) The solution of (2.4) converges uniformly to that of (2.1); this can be shown by a simple extension of the argument of [2]. Further, a trivial generalization of the proof of Lemma 2 of [3] shows that

$$
-a<\frac{u_{i, n}-u_{i-1, n}}{\Delta x}<0, \quad x_{i}<y\left(t_{n+1}\right) .
$$

It is easy to see that this implies (2.3).

Lemma 2. If $x=x(t)$ is the moving boundary for a solution of (1.1), then

$$
b \leq \frac{d x(t)}{d t} \leq b+c a
$$

Proof. This is an immediate consequence of Lemma 1.

Thus, $x(t)$ is a nondecreasing function. Let $t(x)$ be the inverse of $x(t)$ at $\operatorname{such} x$ that the inverse exists and be equal to sup $\{t: x(t)=x\}$ elsewhere.

Lemma 3. If $u(x, t)$ is a solution of (1.1), then $u_{x x}$ and $u_{t}$ are nonnegative.

Proof. The finite difference argument of Lemma 3 of [3] can be adapted to show that the second $x$-difference of $u_{i n}$ are non-negative. From this fact, the above results can be deduced.

Let

$$
F(\xi)=\int_{0}^{\xi} f(z) d z
$$

We can now derive an overall "heat balance" for any solution of (1.1) which will be equivalent to $(1.1 \mathrm{e})$ :

$$
\begin{gathered}
0=\int_{0}^{t} \int_{0}^{x(r)}\left[u_{x x}-f(u) u_{t}\right] d x d r=\int_{0}^{t}\left[u_{x}(x(r), r)-u_{x}(0, r)\right] d r \\
-\int_{0}^{x(t)}[F(u(x, t))-F(u(x, t(x)))] d x .
\end{gathered}
$$


Using (1.1b)-(1.1e),

$$
x(t)=(b+a c) t-c \int_{0}^{x(t)} F(u(x, t)) d x .
$$

Equation (2.8) will be the fundamental relation used in the uniqueness proof.

3. Uniqueness theorem. The theorem is as follows:

TheOREM. The solution of the Stefan problem (1.1) is unique.

Proof. The proof will be broken into two parts. First, we shall show that uniqueness holds in some neighborhood of the origin. Then, it will be shown that $\sup \{T$ : solution is unique for $t \leq T\}=\infty$.

For simplicity, let $d^{*}=b+a c$. Let $x_{1}(t)$ and $u_{1}(x, t)$ be one solution of (1.1), and let $x_{2}(t)$ and $u_{2}(x, t)$ be another. Let

$$
\begin{aligned}
& y(t)=\min \left(x_{1}(t), x_{2}(t)\right), \\
& z(t)=\max \left(x_{1}(t), x_{2}(t)\right) .
\end{aligned}
$$

Then,

$$
\begin{aligned}
& x_{1}(t)=d t^{*}-c \int_{0}^{x_{1}(t)} F\left(u_{1}(x, t)\right) d x, \\
& x_{2}(t)=d t^{*}-c \int_{0}^{x_{2}(t)} F\left(u_{2}(x, t)\right) d x,
\end{aligned}
$$

and

$$
\begin{aligned}
x_{1}(t)-x_{2}(t)= & -c \int_{0}^{y(t)}\left[F\left(u_{1}(x, t)\right)-F\left(u_{2}(x, t)\right)\right] d x \\
& +(-1)^{i} c \int_{v(t)}^{z(t)} F\left(u_{i}(x, t)\right) d x,
\end{aligned}
$$

where $u_{i}$ is the solution defined between $y(t)$ and $z(t)$.

Now, on $x=y(t)$,

$$
\left|u_{1}(y(t), t)-u_{2}(y(t), t)\right| \leq a\left|x_{1}(t)-x_{2}(t)\right|,
$$

by (2.2). It can be shown from the maximum principle for parabolic equations [6] that

$$
\begin{aligned}
\left|u_{1}(x, t)-u_{2}(x, t)\right| \leq a \max & \left|x_{1}(r)-x_{2}(r)\right|, \\
& 0 \leq x \leq y(t), 0 \leq r \leq t,
\end{aligned}
$$

as follows. Let $v(x, t)=u_{1}(x, t)-u_{2}(x, t)$. Then, by subtracting the 
differential equation for $u_{2}$ from that for $u_{1}$, it can be seen that $v$ satisfies the linear parabolic equation

$$
\begin{aligned}
v_{x x} & =f\left(u_{1}(x, t)\right) v_{t}+u_{2, t}(x, t)\left[f\left(u_{1}\right)-f\left(u_{2}\right)\right] \\
& =B_{1}(x, t) v_{t}+u_{2, t} f^{\prime}(\eta(x, t)) v \\
& =B_{1}(x, t) v_{t}+B_{2}(x, t) v .
\end{aligned}
$$

By $(1.1), v_{x}(0, t)=0$. By Lemma $3, u_{2, t} \geq 0$, and by assumption $f^{\prime} \geq 0$; consequently, $B_{2} \geq 0$. Consequently, $v$ is subject to the maximum principle considering only the boundary values on $x=y(t)$ and the required inequality holds.

By the mean value theorem,

$$
F\left(u_{1}(x, t)\right)-F\left(u_{2}(x, t)\right)=f(\xi)\left[u_{1}(x, t)-u_{2}(x, t)\right] .
$$

Consider the interval $0 \leq t \leq T \leq 1$ for the moment. Then, $0 \leq u_{j}(x, t)$ $\leq a d^{*}$ for $0 \leq t \leq T, j=1,2$, by (2.2) and (2.6). Consequently, $|f(\xi)|$ $\leq M$ for some $M$, and

$$
\begin{aligned}
\left|F\left(u_{1}(x, t)\right)-F\left(u_{2}(x, t)\right)\right| & \leq M_{1} \max _{0 \leq r \leq T}\left|x_{1}(r)-x_{2}(r)\right| \\
& 0 \leq x \leq y(t), \\
\mid F\left(u_{1}(x, t)|\leq M| z(t)-x \mid,\right. & y(t) \leq x \leq z(t) .
\end{aligned}
$$

Applying (3.5) to (3.3),

$$
\begin{aligned}
& \left|x_{1}(t)-x_{2}(t)\right| \leq M_{2} y(t) \max _{0 \leq r \leq T}\left|x_{1}(t)-x_{2}(t)\right|+M_{3}\left|x_{1}(t)-x_{2}(t)\right|^{2} \\
& \leq M_{2} y(T) \max _{0 \leq r \leq T}\left|x_{1}(r)-x_{2}(r)\right| \\
& +M_{3} \max _{0 \leq r \leq T}\left|x_{1}(r)-x_{2}(r)\right|^{2}, \\
& 0 \leq t \leq T
\end{aligned}
$$

Therefore, if $A(T)=\max _{0 \leq t \leq T}\left|x_{1}(t)-x_{2}(t)\right|$,

$$
A(T) \leq M_{2} y(T) A(T)+M_{3} A(T)^{2} .
$$

If $A(T)>0$,

$$
1 \leq M_{2} y(T)+M_{3} A(T) .
$$

But, by (2.6), $y(T) \leq d^{*} T$ and $A(T) \leq c a T$; hence, for $T<\left(2 M_{2} d^{*}\right)^{-1}$ $=T_{0}$, we have a contradiction and so $A(T)$ must vanish. Thus, the solution of (1.1) is unique at least for $0 \leq t \leq T_{0}$.

Let $T_{1}=\sup \left\{t: x_{1}(\tau)=x_{2}(\tau), 0 \leq \tau \leq t\right\}$. If $T_{1}=\infty$, then there is nothing left to prove. Assume $T_{1}$ is finite. We shall show that this leads to a contradiction. Let 


$$
A(\Delta t)=\max _{T_{1} \leq t \leq T_{1}+\Delta t}\left|x_{1}(t)-x_{2}(t)\right| .
$$

Again by (2.6), $A(\Delta t) \leq a c \Delta t$. By assumption, $A(\Delta t)>0$ for any $\Delta t>0$. Equation (3.3), of course, still holds, and, as before,

$$
\left|c \int_{y(t)}^{z(t)} F\left(u_{i}(x, t)\right) d x\right| \leq M_{4} A(\Delta t)^{2}, \quad T_{1} \leq t \leq T_{1}+\Delta t .
$$

The approximation of

$$
\int_{0}^{y(t)}\left[F\left(u_{1}(x, t)\right)-F\left(u_{2}(x, t)\right)\right] d x
$$

must be accomplished in another manner. As before,

$$
A(\Delta t) \leq \max _{T_{1} \leq t \leq T_{1}+\Delta t} c \int_{0}^{\nu(t)}\left|F\left(u_{1}(x, t)\right)-F\left(u_{2}(x, t)\right)\right| d x+M_{4} A(\Delta t)^{2} .
$$

Therefore,

$$
\begin{aligned}
1 & \leq \max _{T_{1} \leq t \leq T_{1}+\Delta t} c \int_{0}^{y(t)} \frac{\left|F\left(u_{1}(x, t)\right)-F\left(u_{2}(x, t)\right)\right|}{A(\Delta t)} d x+M_{4} A(\Delta t) \\
& =\max _{T_{1} \leq t \leq T_{1}+\Delta t} c \int_{0}^{y(t)} f(\xi(x, t)) \frac{\left|u_{1}(x, t)-u_{2}(x, t)\right|}{A(\Delta t)} d x+M_{4} A(\Delta t) .
\end{aligned}
$$

For $\Delta t \leq 1, f(\xi)$ is again bounded, since $u_{j}$ is in the strip $T_{1} \leq t \leq T_{1}+1$. Hence,

(3.6) $1 \leq M_{5} \max _{T_{1} \leq t \leq T_{1}+\Delta t} \int_{0}^{y(t)} \frac{\left|u_{1}(x, t)-u_{2}(x, t)\right|}{A(\Delta t)} d x+M_{4} A(\Delta t)$.

Consider $v(x, t)=u_{1}(x, t)-u_{2}(x, t)$, as before. As $v\left(x, T_{1}\right) \equiv 0$ and $|v(y(t), t)| \leq a A(\Delta t)$ for $T_{1} \leq t \leq T_{1}+\Delta t,|v(x, t)| \leq a A(\Delta t)$ for $0 \leq x \leq y(t)$, $T_{1} \leq t \leq T_{1}+\Delta t$ by the maximum principle. Hence,

$$
\int_{y\left(T_{1}\right)}^{y(t)} \frac{|v(x, t)|}{A(\Delta t)} d x \leq a\left[y(t)-y\left(T_{1}\right)\right] \leq a d^{*} \Delta t .
$$

This leaves the integral from 0 to $y\left(T_{1}\right)$ to consider. Consider the auxiliary problem

$$
\begin{aligned}
v_{x x}^{*} & =B_{1} v_{t}^{*}+B_{2}{ }^{*}, & 0<x<y\left(T_{1}\right), T_{1}<t, \\
v^{*}\left(x, T_{1}\right) & =0, & 0<x<y\left(T_{1}\right) \\
v_{x}^{*}(0, t) & =0, & t>0, \\
v^{*}\left(y\left(T_{1}\right), t\right) & =1, & t>0,
\end{aligned}
$$


where the $B_{1}$ and $B_{2}$ are those derived in (3.4). The existence of $v^{*}$ is guaranteed by [5]. Then, by the maximum principle,

$$
\frac{|v(x, t)|}{a A(\Delta t)} \leq v^{*}(x, t) \leq 1, \quad 0 \leq x \leq y\left(T_{1}\right), T_{1} \leq t \leq T_{1}+\Delta t .
$$

As for $0 \leq x<y\left(T_{1}\right), \lim _{t \downarrow T_{1}} v^{*}(x, t)=0$, Lebesgue's bounded convergence theorem implies that

$$
\lim _{t \downarrow T_{1}} \int_{0}^{y\left(T_{1}\right)} v^{*}(x, t) d x=0 .
$$

Consequently,

$$
\max _{T_{1} \leq t \leq T_{1}+\Delta t} \int_{0}^{y\left(T_{1}\right)} \frac{|v(x, t)|}{A(\Delta t)} d x \rightarrow 0
$$

as $\Delta t \rightarrow 0$. As (3.7) and (3.9) contradict (3.6), the proof of the uniqueness theorem is completed.

\section{REFERENCES}

1. A. Datzeff, Sur le problème linéaire de Stefan, II, Annuaire de l'Université de Sofia. I. Faculté Physico-Mathématique. Livre 1 vol. 46 (1950) pp. 271-325.

2. J. Douglas, Jr., On the numerical integration of quasi-linear parabolic equations, Pacific Journal of Mathematics vol. 6 (1956) pp. 35-42.

3. J. Douglas, Jr. and T. M. Gallie, Jr., On the numerical integration of a parabolic differential equation subject to a moving boundary condition, Duke Math. J. vol. 22 (1955) pp. 557-572.

4. G. W. Evans, II. A note on the existence of a solution to a problem of Stefan, Quarterly of Applied Mathematics vol. 9 (1951) pp. 185-193.

5. M. Gevrey, Sur les equations aux dérivées partielles du type parabolique, J. Math. Pures Appl. Series 6 vol. 9 (1913) pp. 305-471.

6. L. Nirenberg, $A$ strong maximum principle for parabolic equations, Communications on Applied Mathematics, vol. 6 (1953) pp. 167-177.

7. G. Sestini, Esistenza di una soluzione in problemi analogli a quello di Stefan, Rivista di Matematica della Università di Parma vol. 3 (1952) pp. 3-23.

Humble Oil and Refining Co. 